\title{
Philosophiques
}

\section{Contingence ou inévitabilité des résultats de notre science?}

\section{Léna Soler}

Volume 33, numéro 2, automne 2006

URI : https://id.erudit.org/iderudit/013887ar

DOI : https://doi.org/10.7202/013887ar

Aller au sommaire du numéro

Éditeur(s)

Société de philosophie du Québec

ISSN

0316-2923 (imprimé)

1492-1391 (numérique)

Découvrir la revue

Citer cet article

Soler, L. (2006). Contingence ou inévitabilité des résultats de notre science?

Philosophiques, 33(2), 363-378. https://doi.org/10.7202/013887ar d'utilisation que vous pouvez consulter en ligne.

https://apropos.erudit.org/fr/usagers/politique-dutilisation/ 


\title{
Contingence ou inévitabilité des résultats de notre science?
}

\author{
LÉNA SOLER \\ IUFM de Lorraine, Archives H. Poincaré (LPHS) \\ Lena.soler@univ-nancy2.fr
}

\section{Introduction}

L'article discute certains aspects de la question de savoir si une partie du tout de notre physique peut être détachée des contingences de l'histoire humaine et élevée au statut de résultat inévitable. Cette réflexion circonscrite gagne à être au préalable replacée dans le contexte plus large d'où elle tire un sens et une portée spécifiques, à savoir, une recherche visant à élaborer un modèle dit «symbiotique» de développement scientifique, inspiré notamment des travaux de Ian Hacking (Hacking, 1992) et de Andrew Pickering (Pickering, 1995). Une telle mise en contexte permettra de mieux saisir les enjeux de la question, ainsi que la nature de son lien essentiel aux problèmes du holisme épistémologique et de la sous-détermination. Dans un second temps, nous nous pencherons sur les caractères que devrait présenter une physique alternative pour donner lieu à un contingentisme épistémologiquement conséquent, puis nous examinerons les justifications dont peut espérer disposer l'inévitabilisme.

\section{Le symbiotisme et le holisme impliquent-ils le contingentisme ?}

Adopter un modèle symbiotique de développement scientifique oblige-t-il à endosser le contingentisme? Cette question, qui ne sera pas approfondie dans l'article, constitue pourtant l'arrière-plan des réflexions qui vont suivre. Esquissons-en la teneur et les enjeux.

En première approche, le contingentisme affirme la possibilité d'une science aussi performante et progressive que la nôtre mais radicalement autre en teneur, notamment sur le plan ontologique. Hacking nomme la position antagoniste «inévitabilisme ${ }^{1}$. Selon l'inévitabilisme, toute science aussi performante et progressive que la nôtre qui se serait posé les mêmes questions que la nôtre devait inévitablement aboutir à des réponses fondamentalement semblables aux nôtres.

Le recours au vocable de l' « inévitable » plutôt qu'à celui du "nécessaire » est justifié par la possibilité - que personne ne conteste - que les êtres humains auraient pu, mus par d'autres intérêts et idéaux, ne jamais chercher à élaborer quoi que ce soit qui ressemble à de la physique, ou encore vouloir

1. Dans l'article de Hacking (2000), qui constitue l'une des sources majeures de la présente réflexion. Voir aussi Hacking (1999), pp. 70 et suivantes. 
faire de la physique mais échouer à développer une physique aussi performante et progressive que la nôtre.

Un représentant emblématique du contingentisme est le sociologue des sciences constructivistes Andrew Pickering (Pickering, 1995). Un représentant emblématique de l'inévitabilisme est le physicien Stephen Weinberg ${ }^{2}-$ réaliste comme la plupart des scientifiques. Pickering a développé ses positions philosophiques en s'appuyant essentiellement sur le cas de la physique des particules (Pickering, 1984), et Weinberg a été un acteur important du développement de cette physique. C'est aussi en référence à ce cas particulier qu'a été conduite la réflexion qui va suivre, même si aucune mention explicite approfondie n'en sera faite dans l'article ${ }^{3}$.

Les associations qu'exemplifient ici Pickering et Weinberg, entre constructivisme et contingentisme d'un côté, réalisme et inévitabilisme de l'autre, sont empiriquement fréquentes et ont des raisons d'être conceptuelles. Pourtant, d'un point de vue logique, la problématique «contingentisme ou inévitabilisme » ne coïncide nullement avec la problématique « réalisme ou constructivisme anti-réaliste » - même s'il existe entre ces quatre pôles tout un réseau de relations en attente d'être systématiquement explicitées (des éléments partiels sont proposés plus bas concernant les relations entre réalisme et inévitabilisme). En outre, à mon avis, la première opposition a tout intérêt à être nettement distinguée de la seconde et examinée pour elle-même. Or c'est rarement le cas actuellement dans la littérature spécialisée - en dépit des travaux récents de Hacking qui poussent fortement dans cette direction ${ }^{4}$. En fait, l'alternative " contingentisme ou inévitabilisme » n'est pas une opposition majeure relativement autonome bien identifiée, et n'est que rarement discutée à l'état isolé en tant que chapitre indépendant des réflexions contemporaines sur la science - contrairement à la dichotomie réalisme/antiréalisme scientifique. Même si la question du contingentisme affleure souvent dans les débats consacrés au réalisme, le point focal de ceux-ci reste le rapport des conceptualisations humaines au Réel, et non

2. (Weinberg, 1996). Voir aussi les propos du physicien Sheldon Glashow rapportés dans Hacking, $(2000,66)$.

3. Cette précision concernant l'exemple physique de référence est motivée par une réflexion que m'a adressée Ian Hacking, à savoir que des analyses adossées à la physique des particules ne sont peut-être pas généralisables à toute physique, et, en l'occurrence, que le contingentisme, qui peut apparaître convaincant à certains esprits dans le cas d'une physique très théorique et complexe comme l'est la physique des particules, l'est peut-être beaucoup moins dans le cas d'autres secteurs de la physique. La remarque vise donc à laisser ouverte la question de cette généralisation.

4. Quand c'est le cas, c'est souvent dans un contexte où sont discutées les réflexions de Hacking sur la question - lesquelles distinguent explicitement le contingentisme et le réalisme et érigent le contingentisme en problème autonome (Hacking 1999, 2000). Voir par exemple Franklin (2002, section 5). La question du contingentisme est adressée aux physiciens Bernard d'Espagnat et Hervé Zwirn, qui y apportent des réponses substantiellement différentes dans Soler (2006a). 
celui de la contingence/inévitabilité de certains secteurs de ces conceptualisations. Bref, la question du réalisme monopolise l'attention et, ce faisant, occulte l'intérêt propre de la question de la contingence.

Cet intérêt tient notamment au fait que certaines formes de contingentisme offrent des candidates de conceptions de la science intéressantes: intéressantes en ce que, à la fois elles sont moins fortes que le réalisme correspondantisme - lequel, comme l'on sait, exige beaucoup et est sujet à des critiques de l'avis de nombreux auteurs rédhibitoires —, sans pour autant brader la rationalité et le progrès scientifique. Les conceptions que nous choisissons de réunir sous le qualificatif de "symbiotique ", en particulier, sont sous-tendues par un schéma de développement scientifique susceptible de porter un contingentisme de cette espèce. Esquissons donc à présent les traits fondamentaux d'un tel schéma.

Dans un modèle de type symbiotique, la physique consiste à « faire tenir ensemble " une multitude d'éléments intervenant dans les pratiques expérimentales et théoriques. La nature des éléments pertinents est source de controverses, mais une hypothèse consensuelle fondamentale est qu'ils relèvent de genres hétérogènes beaucoup plus diversifiés que ceux mis en avant par la tradition dite «linguistique " (pas seulement des énoncés, donc, mais aussi des objets matériels, des marques résultant des expériences, des savoir-faire, etc.). Sur une ligne diachronique, il y a co-maturation des divers ingrédients; et dans certains plans synchronique, émergent de plus ou moins bonnes costabilisations : des unités holistiques relativement stables, que nous appelons "symbioses scientifiques ». Les symbioses nomment des associations d'éléments suffisamment rigides et verrouillées pour constituer ce qui est communément décrit comme l'opérativité ou l'efficacité caractéristique de la physique moderne. Le progrès consiste dans l'obtention effective de symbioses toujours plus puissantes.

Le schéma structurel qui sous-tend l'idée d'un progrès «symbiotique " des sciences est essentiellement holistique. Le tout prime sur la partie, l'équilibre global est au-dessus des ingrédients. L'élément est en effet expliqué à partir de sa place au sein d'un tout (réseau d'interactions plutôt que causalités linéaires). Il n'y a pas de type d'ingrédient prédéterminé qui, a priori et toujours, pilote seul, de manière autonome, le processus (théorie ou résultat expérimentaux par ex.). Il y a stabilisation mutuelle, ajustement réciproque. C'est la qualité relative des équilibres globaux effectivement atteints qui détermine les évaluations d'adéquation empirique faites par les praticiens et sous-tend l'affirmation d'un progrès sur ce plan.

Plus : joint à la diversité des types d'ingrédients qui constituent les pratiques scientifiques, le schéma conduit à admettre, selon l'expression de Hacking, une "extension de la thèse de Duhem » (Hacking, 1992): en quête de bonnes symbioses, les praticiens peuvent jouer sur beaucoup plus de genres d'items que ne le soutient la thèse de Duhem-Quine communément interprétée comme un jeu entre énoncés. 
Ces traits fondamentaux du modèle symbiotique semblent impliquer au moins une "certaine dose» de contingentisme. En effet, le fait que cette symbiose particulière émerge effectivement à un moment donné de l'investigation, dépend évidemment des éléments effectivement présents au moment en question (puisqu'une symbiose est une co-stabilisation). En outre, ce fait n'est pas univoquement déterminé par ces éléments (à cause de la thèse de Duhem, en l'occurrence étendue). En chaque point de la trajectoire historique, on peut imaginer que d'autres ingrédients auraient pu être présents, et aussi qu'étant donné la même situation initiale, d'autres ajustements mutuels auraient pu être trouvés: on a une histoire des sciences dépendante du chemin suivi (ce qui suit dépend essentiellement de ce qui précède); et on a en même temps une histoire des sciences ouverte (ce qui suit n'est pas univoquement déterminé par ce qui précède). La physique aurait pu se stabiliser de multiples manières différentes (et nous pouvons former l'idée d'alternatives de types multiples ${ }^{5}$ ).

Le symbiotisme implique donc de la contingence, et peut s'accompagner d'un contingentisme radical, comme c'est le cas notamment chez Pickering. Mais implique-t-il pour autant que tout soit contingent dans notre science? Hacking (2000), par exemple, semble considérer que non: il assume en effet explicitement un certain inévitabilisme (certes modéré), alors même qu'il défend par ailleurs un modèle de type symbiotique.

La question est délicate et requiert une discussion approfondie. Elle prend une forme particulière et revêt une importance spécialement cruciale dans le cadre d'un modèle symbiotique - surtout dans le cadre d'une recherche qui, comme la nôtre, vise à isoler une sorte de minimum suffisamment consensuel de progrès scientifique - , et mise sur le modèle symbiotique pour incarner ce plus petit dénominateur commun (ce qui ne saurait être le cas si le symbiotisme rend strictement impossible de soustraire une partie de notre science à la modalité contingente - car alors aucun réaliste ne sera prêt à l'admettre en tant que minimum de progrès). Mais la question importe bien au-delà du cadre symbiotique et de l'objectif particulier qui vient d'être mentionné. En effet, une question structurellement similaire se pose à tout penseur des sciences qui admet le caractère intenable de l'atomisme épistémologique et le fonctionnement jusqu'à un certain point holistique des pratiques scientifiques humaines. Or il est pour le moins difficile de ne pas l'admettre.

5. Une espèce concevable d'alternative serait par exemple une physique qui se serait développée à partir d'instruments de mesure disjoints de ceux de notre physique. Pickering et Hacking considèrent cette possibilité, le premier la repérant comme une "incommensurabilité machinique » (Pickering, 1995, section 6.2), le second comme une «incommensurabilité littérale » (les deux physiques considérées n'auraient, littéralement, pas de mesures physiques en commun) (Hacking, 1992). Le sens et les enjeux d'une telle éventualité, ainsi que la pertinence de sa désignation en terme d'incommensurabilité, sont discutés dans Soler (2005 et 2006b). 


\section{Types de physiques alternatives susceptibles de donner lieu à un contingentisme épistémologiquement conséquent}

Examinons à présent les types de physiques alternatives à notre physique qui, si elles pouvaient advenir, incarneraient la possibilité d'un contingentisme non inoffensif d'un point de vue épistémologique.

Cette investigation est requise, car il y a des sens en lesquels le contingentisme est lui-même - pourrait-on dire en cultivant le paradoxe inévitable. Pour prendre un cas extrême, imaginons que d'autres physiciens se soient posé des questions disjointes des nôtres et qu'ils aient abouti à des réponses disjointes des nôtres. Une telle possibilité correspond bien à une physique alternative radicalement différente de la nôtre, puisque sans intersection du point de vue des résultats. Toutefois, cette possibilité est épistémologiquement inoffensive tant que la disjonction de fait n'est pas une incompatibilité en droit. Sans cette clause d'incompatibilité, elle n'inquiète ni le réaliste ni l'inévitabiliste. Elle ne fait à leurs yeux qu'illustrer cette évidence, qu'un objet d'étude n'est, pour les créatures finies que sont les êtres humains, jamais investiguable d'un seul coup sous toutes ses facettes, mais toujours seulement petit à petit, selon un ordre de priorités et des chemins qui auraient pu être autres. Ici la contingence apparaît inoffensive, du fait qu'elle est toute reportée sur l'ordre et les manières d'une investigation dont les résultats sont quant à eux maintenus fixes.

Alors, que faut-il donc pour que le contingentisme devienne épistémologiquement conséquent ? La réponse qui va être explicitée est la suivante : il faut qu'il y ait, entre notre physique et les physiques alternatives invoquées, des différences irréconciliables sur fond de parentés significatives.

\section{Deux physiques alternatives présentant des parentés significatives}

Commençons par justifier et spécifier l'exigence de parentés significatives. Elle est justifiée par la remarque suivante : l'idée d'accomplissements radicalement différents de ceux de notre physique (disons $\mathrm{P}_{\text {eff }}$ comme "effective») ne soulève un problème épistémologique, que si les accomplissements en question sont issus d'une discipline (disons $\mathrm{P}_{\text {pot }}$ comme "potentielle ») qui puisse être considérée comme une rivale authentique de notre physique. En d'autres termes, il faut que $\mathrm{P}_{\text {pot }}$ soit elle-même une physique digne de ce nom.

Cette condition est loin d'être triviale. Sans pouvoir l'approfondir ici, indiquons seulement qu'elle se laisse analyser en deux exigences relevant de deux plans conceptuellement distincts quoiqu'interactifs en pratique, celui de l'objet et celui de la méthode.

a) L'objet visé par les deux recherches comparées doit pouvoir être considéré comme "suffisamment le même». L'exigence de l'identité objectale est requise pour juger que l'on a bien affaire à deux physiques, pour reconnaître en $\mathrm{P}_{\text {pot }}$ une physique plutôt que, par ex., une biologie ou une autre espèce quelconque de pratique. 
b) Les deux pratiques comparées doivent pouvoir être considérées comme deux disciplines suffisamment semblables. L'exigence de l'identité disciplinaire est requise pour juger que l'on a bien affaire à deux physiques dignes de ce nom: pour reconnaître en $\mathrm{P}_{\text {ot }}$ une physique authentique et non une pseudo-science n'ayant de physique que le nom.

La condition a impose un minimum de commensurabilité conceptuelle entre $\mathrm{P}_{\text {eff }}$ et $\mathrm{P}_{\text {pot }}$, au moins quant à la manière dont toutes deux conçoivent ce qu'est la réalité physique en général par opposition à d'autres ordres de réalités (biologique, psychologique, etc.).

Corrélativement, la condition $b$ impose un minimum de commensurabilité méthodologique au sens large: un minimum de normes et de valeurs partagées à propos de ce qu'est une bonne physique, de ce qu'est un argument ou une preuve physique digne de ce nom, etc.

\section{Deux physiques alternatives présentant des différences irréconciliables}

Ayant considéré l'exigence de parentés significatives, tournons-nous à présent vers l'exigence opposée, celle des types de différences qui doivent valoir entre $\mathrm{P}_{\text {eff }}$ et $\mathrm{P}_{\text {pot }}$.

Pour être épistémologiquement conséquentes, ces différences doivent être non juxtaposables, non additionnables : elles doivent être des incompatibilités, ou au moins des tensions fortes de nature à spécifier.

Nous en distinguerons deux espèces : les incompatibilités descriptives et les incompatibilités axiologiques ${ }^{6}$.

\subsection{Incompatibilités descriptives}

Le plan descriptif est ici entendu en un sens très large: il inclut tous les énoncés explicites, en mots ou en symboles mathématiques, qui peuvent avoir une prétention descriptive et qui médiatisent communément, à quelque échelle que ce soit, les rapports des praticiens avec l'objet visé; et il inclut aussi tout ce qui est implicitement assumé ou présupposé par les acteurs à propos de ce qu'est l'objet d'étude et de la manière dont il se comporte.

Sans pouvoir développer, donnons quelques exemples de types concevables d'incompatibilités descriptives entre notre physique $\mathrm{P}_{\text {eff }}$ et une physique alternative contrafactuelle $\mathrm{P}_{\text {pot }}$ :

- incompatibilité entre les entités et processus fondamentaux mobilisés par les théories de haut niveau;

- incompatibilité entre les éléments ayant statut de faits expérimentaux;

6. Il n'est peut être pas inutile de noter que les registres du descriptif et du normatif entretiennent des liens étroits et à double sens au sein d'une même pratique. Si leur distinction est clarifiante d'un point de vue analytique, elle ne doit pas suggérer une séparation in concreto. 
- incompatibilité entre les formes d'équations mathématiques fondamentales manipulées;

- incompatibilité entre les valeurs des grandeurs assimilées à des constantes fondamentales.

Dans les discussions qui touchent au caractère contingent des résultats de notre science, quelques cas particuliers relevant de ces types reviennent presque immanquablement, qui exemplifient de manière frappante des espèces d'accomplissements que la plupart des physiciens ne peuvent pas ne pas croire inévitables. Tel est le cas du second principe de la thermodynamique, des équations de Maxwell, ou encore de la constance de la vitesse de la lumière et de l'ordre de grandeur que notre physique indique pour cette constante (Hacking, 2000).

Pour revenir à la caractérisation générale des différences, distinguons encore, au sein des incompatibilités descriptives, deux sous-espèces : les contradictions et les incommensurabilités sémantiques-taxonomiques.

Le cas de la contradiction présuppose une commensurabilité conceptuelle importante : au moins la possibilité de ré-exprimer dans un seul et même langage commun quelque chose de suffisamment substantiel des descriptions explicites ou des assomptions implicites mobilisées par les praticiens de $\mathrm{P}_{\text {eff }}$ et $\mathrm{P}_{\text {pot: }}$.

Le cas des incommensurabilités sémantiques-taxonomiques est plus complexe à appréhender. Disons que la différence mise en jeu touche non plus le niveau des valeurs de vérité, mais celui des ressources même de l'expression-description. Plus précisément, les incommensurabilités sémantiquestaxonomiques présupposent le recours à des structures lexicales ou symboliques localement non isomorphes coordonnées à des réseaux conceptuels localement non superposables - certaines choses s'avérant alors dicibles d'un côté et non de l'autre?

\subsection{Incompatibilités axiologiques}

Venons-en à présent aux incompatibilités axiologiques.

Sur fond du minimum de commensurabilité méthodologique requis pour avoir affaire à deux physiques authentiques en un sens suffisamment proche pourraient survenir des incommensurabilités normatives locales : par exemple, des incompatibilités concernant la fiabilité ou la pertinence de telle ou telle procédure expérimentale particulière.

De telles différences sont épistémologiquement conséquentes, dans la mesure où les incompatibilités locales relatives à la fiabilité et à la pertinence

7. On trouvera une description d'ensemble du problème de l'incommensurabilité et de ses transformations depuis son introduction en philosophie des sciences au début des années soixante, ainsi qu'une bibliographie actualisée conséquente sur le sujet, dans Soler (2004). Voir aussi Hoyningen (1989) et Sankey (1994). 
des moyens de la recherche ont toutes les chances d'induire des incompatibilités au niveau des résultats de cette recherche (dans l'exemple, des incompatibilités au niveau de ce qui fait figure de résultat expérimental authentique par opposition à des artéfacts ou à du bruit). Sous l'hypothèse d'une commensurabilité méthodologique globale suffisante ${ }^{8}$, les incompatibilités axiologiques locales sont donc finalement conséquentes en tant que sources d'incompatibilités descriptives, ces dernières étant, en fin de compte, celles qui importent ultimement d'un point de vue épistémologique.

Voilà pour l'inventaire rapide des types d'incompatibilités qui, si elles pouvaient survenir, correspondraient à un contingentisme potentiellement offensif d'un point de vue épistémologique. Examinons à présent la question des arguments mobilisés dans le débat contingentisme/inévitabilisme.

\section{Arguments en faveur de l'inévitabilisme}

Quand on considère ce débat s'impose à première vue presque inévitablement l'impression que la charge de la preuve revient aux contingentistes.

Toutefois, cette impression s'avère à l'examen trompeuse : la charge de la preuve n'est au moins pas plus dans le camp des contingentistes que dans celui des inévitabilistes. Telle est la position que je vais m'employer à rendre plausible.

Une telle position doit se situer par rapport au fait que l'intuition, et en premier lieu l'intuition des praticiens des sciences eux-mêmes, soutient fortement l'inévitabilisme. L'expérience quotidienne des physiciens est incontestablement dominée par le sentiment que des contraintes extérieures puissantes s'exercent sur l'élaboration théorique et expérimentale: des contraintes qui laissent peu de marge de manœuvre, voire qui, au fil des investigations, imposent la bonne réponse, l'unique solution.

Aussi la plupart des scientifiques sont-ils, à l'instar de Weinberg, inévitabilistes et réalistes. Face aux contingentistes, ils brandissent ce que

8. Si on lève la condition d'une commensurabilité méthodologique globale suffisante, par exemple si on juge plausible la possibilité qu'à partir d'un certain état initial de notre physique auraient pu se développer d'autres pratiques estimées scientifiques du point de vue des acteurs (fiables en termes d'établissement de résultats crédibles), mais n'ayant plus grand-chose à voir avec les démarches et les normes qui constituent notre science, alors, s'introduit un enjeu épistémologique supplémentaire, conceptuellement distinct de celui des incompatibilités descriptives. Cet enjeu correspond à une question controversée déjà posée à propos de notre physique considérée seule : celle du degré d'universalité de la démarche scientifique, et plus généralement celle d'un étalon non problématique des jugements humains. C'est donc tout le chapitre du relativisme qui s'ouvre ici. Mais cette question des incommensurabilités axiologiques susceptibles d'exister entre deux pratiques qui prétendraient l'une et l'autre incarner le paradigme de la science ne se pose pas dans le cadre du problème du contingentisme/inévitabilisme tel qu'il a été délimité ici. En effet, ici le problème consiste à discuter la possibilité de deux physiques aussi progressives et performantes l'une que l'autre mais néanmoins coordonnées à des résultats incompatibles. Or la clause d'une progressivité et performativité d'égale dignité exige un minimum significatif de commensurabilité axiologique globale. 
Hacking (2000) appelle la put-up-or-shut-up answer: "montre un tel développement scientifique alternatif, ou tais-toi». L'idée d'une physique aussi performante et progressive que la nôtre qui serait coordonnée à des résultats incompatibles - en l'un des sens répertoriés plus haut -, leur apparaît au mieux extrêmement peu crédible, et le plus souvent ridicule, absurde, voire scandaleuse ou obscène.

À les suivre et à les considérer comme des interlocuteurs à convaincre, la première tâche à accomplir consisterait à rendre le contingentisme plausible — c'est-à-dire à conférer quelque plausibilité au moins à certaines des potentialités décrites plus haut.

Ce n'est pourtant pas ce que nous ferons ici. À l'inverse, nous nous proposons de demander des comptes à l'inévitabiliste, en posant la question : audelà des intuitions et du vécu des praticiens, quels arguments peut-on invoquer en faveur de l'inévitabilisme?

\section{Le réalisme comme justification de l'inévitabilisme ?}

La première justification qui vient à l'esprit c'est, bien entendu, la pression du réel, les contraintes exercées par le référent. Plus : si ces contraintes doivent "à terme" réduire à l'unité les réponses à une question scientifique «bien posée ", ce ne sont pas seulement des contraintes, mais quelque chose comme une causalité stricte (un déterminisme univoque), que doit exercer la "chose en soi " (étant donné ce qu'est l'homme et ce que sont les formes de la connaissance humaine).

Ici, le réalisme scientifique sous-tend et explique l'inévitabilisme.

Notons que le réalisme en question n'est pas nécessairement correspondantiste. On n'est en effet pas obligé de considérer les réponses retenues par les physiciens comme des descriptions littérales fidèles de fragments d'une réalité indépendante pré-structurée. Il est seulement requis que la pression de cette réalité soit suffisamment forte pour départager les diverses propositions humaines et imposer une unique solution.

Et encore, on peut assouplir cette exigence. Au nom du fait que les réponses humaines ne sont jamais ultimes et peuvent toujours être précisées, on peut en effet se contenter d'exiger que le monde physique impose, non pas une unique réponse à une question scientifique bien posée dotée de réponse, mais une unique famille de solutions non problématiquement compatibles entre elles.

Il est hors de question de discuter ici les difficultés des positions de ce type - difficultés qui recouvrent, outre celles, bien connues, inhérentes aux principales variétés de réalisme, celles de donner un sens à des clauses telles que " une réponse unique à terme», " une question physique bien posée» ou encore " une famille de réponses non problématiquement compatibles entre elles ».

Je me contenterai de retourner au réaliste-inévitabiliste un message symétrique au put-up-or-shut-up qu'il adresse au contingentiste: "Montre, 
dans les pratiques scientifiques contemporaines, cette part de réalité indépendante censée déterminer univoquement les résultats, ou tais-toi. »

Quand on examine la physique, quel est le candidat le plus susceptible d'être identifié à un porteur de réalité indépendante ? La réponse paraît être : les «marques» (entendues comme observations minimalement interprétées ou degré zéro du phénomène) qui résultent d'expériences estimées fiables; par ex., dans le cas de la physique des particules, les photographies prises dans des chambres à bulles.

Or ces marques, personne ne le conteste aujourd'hui, sont loin d'être de purs « extraits de réel ». Elles sont en effet fortement tributaires, dans leur existence, leur pertinence et leur sens, des machines, des techniques et des assomptions implicites et explicites mobilisées dans les pratiques scientifiques. Plus : même à les assimiler à des données inéliminables, il est fort difficile de soutenir qu'elles imposent à elles seules une famille restreinte de conceptions à propos du monde physique.

À l'appui de ce dernier point, les contingentistes invoquent une version ou une autre, plus ou moins forte selon les cas, de la thèse Duhem-Quine (voir partie I). Pickering, notamment, insiste sur l'extrême plasticité des pratiques scientifiques (Pickering, 1995). L'idée est que les types d'éléments susceptibles de jouer le rôle de "variables d'ajustement » dans la quête pour l'obtention de bonnes adéquations entre théorie et expérimentation sont hétérogènes et nombreux (d'autant plus que les pratiques scientifiques sont complexes, comme dans le cas de la physique des particules) : les physiciens peuvent modifier ou ignorer non seulement des énoncés (à propos de la réalité physique inobservable, des instruments de mesure, des données expérimentales, etc.), mais aussi, entre autres, des objets matériels, des savoir-faire, des procédures pragmatiques d'approximation, des techniques d'analyse de données... Si donc les praticiens peuvent, à chaque moment de l'histoire des sciences, " jouer » sur de très nombreux éléments hétérogènes pour satisfaire l'idéal régulateur commun d'ajustement optimal, alors, même à conférer aux marques résultant des expériences le statut privilégié de données irrécusables à intégrer absolument, restent toujours en principe possibles plusieurs ajustements satisfaisants à chaque étape - même si en pratique un seul est effectivement proposé, ou plusieurs sont proposés mais un seul, celui qui est comparativement estimé le meilleur, est finalement retenu comme «le bon».

Pour citer un passage significatif de Pickering allant dans ce sens :

[...] my analysis of practice [...] points to a situatedness and path dependence of knowledge production. On the one hand, what counts as empirical or theoretical knowledge at any time is a function not just of how the world is but of the specific material-conceptual-disciplinary-social-etc. space in which knowledge is situated. On the other hand, what counts as knowledge is not determined by the space in which it is produced. As I have emphasized, one needs also to take into account the contingencies of practice, the precise route that practice takes through that space. The contingent tentative fixing of modelling 
vectors, the contingent resistances that arise, the contingent formulation of strategies of accommodation, the contingent success or failure of these - all of these structure practice and its products.

Si l'on admet cette analyse et que l'on veut néanmoins soutenir le réalisme correspondantiste - ou au moins l'unicité d'une famille de résultats scientifiques inévitables:

It seems necessary to suppose that the situatedness and path dependance [...] somehow (and sometimes) wash out, so that scientific knowledge eventually converges on a mirroring relation to nature, independently of where scientific practice starts and whatever direction it sets off in. I can only note that nothing in my analysis points to such an erasure of situatedness and path dependence (Pickering, 1995, 185).

\section{Une autre justification de l'inévitabilisme? L'argument de l'auto-consistance de la physique}

Peut-on invoquer, en faveur de l'inévitabilisme, un autre type d'argument que le réalisme?

a) Nous n'en voyons qu'un autre possible, que nous baptiserons pour faire vite «l'argument de l'auto-consistance de la physique». Il consiste en substance à rapporter le sentiment de détermination des physiciens à un réseau de contraintes "constitutives » au sens de " essentielles à l'identité de notre physique ", au sens de "engendrées par les caractères propres de la physique telle qu'elle est conçue et pratiquée à l'époque moderne ».

L'idée est que la nature spécifique de la pratique et des accomplissements de la physique est telle que, dans chaque état de développement, s'exerce de fait sur les physiciens un système de contraintes, qui restent à caractériser en détail mais dont au moins une fraction irréductible ne sera reconnue par personne comme "purement externe ", et qui, de plus, pèsent assez fortement et uniformément sur les praticiens pour agir dans le sens d'une réduction très importante de leur marge de manœuvre et imposer dans les faits une famille restreinte de réponses aux questions posées.

La question de la nature, de l'origine et du mode d'action des contraintes mises en jeu appelle une analyse approfondie. Disons seulement ici qu'il faudrait prendre en considération au moins quatre caractéristiques hétérogènes - partiellement interdépendantes et ci-dessous listées dans un ordre n'ayant pas vocation à refléter une hiérarchie - de notre physique.

Tout d'abord, la volonté déterminée et active des praticiens de mettre leurs propositions à l'épreuve d'un Autre-de-l'homme (le Réel) - trait essentiel de toute discipline scientifique. Dans la physique telle qu'elle est pratiquée actuellement, cet Autre-de-l'homme est 
considéré se manifester par le biais de l'expérimentation systématique et des "marques" qui en résultent. L'exigence commune d'être spécialement attentif, voire de se soumettre aux manifestations du Réel, devient ainsi celle d'intégrer le plus harmonieusement possible ce qui fait figure de résultat expérimental.

Cette intégration n'est pas anarchique, ses modalités ne sont pas quelconques : elle est fortement normée. L'adhésion des acteurs à des idéaux régulateurs très largement partagés, tels ceux de cohérence, de généralité, ou d'économie maximale — qui ne sont pas propres à la physique mais qui y agissent de manière déterminante - , vient limiter l'espace en principe infini des possibilités conceptuelles. La volonté unanime des physiciens d'écouter la voix du Réel se traduit alors concrètement par des tentatives (de fait toujours plus réussies) pour «mettre en consilience » un maximum de marques résultant d'expériences avec un minimum d'ingrédients théoriques.

La nature et l'opérativité des normes qui régulent effectivement ces tentatives sont redevables de certains caractères de notre éducation scientifique. Cette éducation présente notamment la particularité d'être assez fortement uniformisante: tout au long du processus d'apprentissage des connaissances théoriques et des compétences pratiques qui constituent les acquis de référence dans un état de développement, elle installe des habitus relativement homogènes quant aux catégories, aux problèmes, aux arguments et aux solutions qui sont authentiquement physiques et qui ne le sont pas. Ce faisant, elle inculque des normes de scientificité plus ou moins générales qui peuvent être implicites et floues mais qui néanmoins opèrent (l'expérimentation constitue le paradigme de la preuve, les forces à distance sont/ne sont pas un modèle admissible en physique...), en même temps qu'elle confère des contenus intuitifs particuliers aux principes généraux de cohérence, de généralité, d'économie, etc. Une fois intériorisés, ces éléments agissent comme des contraintes fortes, communes à un ensemble plus ou moins large de praticiens, et qui s'imposent "de l'extérieur" aux individus de cet ensemble.

Enfin, il faudrait intégrer à la discussion de l'argument d'autoconsistance le type spécifique de dynamique qui sous-tend le progrès de notre physique. Ce progrès manifeste (à un certain niveau tout au moins) une cumulativité d'un certain type, qui correspond, non pas à la simple addition d'éléments autonomes, mais à une construction de nouvelles strates à la fois largement non destructive et fortement tributaire des anciennes. On peut concevoir une science qui progresserait selon d'autres schémas; mais, dans notre physique, l'édification et la légitimation des nouveaux accomplissements, à la 
fois s'effectuent en préservant une multitude d'anciens acquis (conformément au principe d'économie) et s'appuient constitutivement sur nombre d'entre eux (qui fonctionnent souvent comme des «boîtes noires» techniques ou théoriques).

Il résulte de ce processus "en escalier ", que, sur le plan diachronique, le présent de la science est jusqu'à un certain point dépendant de son passé, et que dans chaque coupe synchronique des liens complexes de dépendance réciproque existent entre les divers ingrédients constitutifs de la physique (voire aussi d'autres sciences). Au sein du tout de notre physique, diverses parties se soutiennent mutuellement selon des configurations complexes.

Cette situation est une puissante source de contraintes pour les physiciens. Le réquisit de conserver un maximum d'acquis antérieurs dans le processus d'intégration du nouveau, joint au fait que toucher à une partie (théorique, expérimentale, technique...) conduit à toucher à beaucoup d'autres, introduisent un réseau de contraintes en général fort difficile à satisfaire. Fort difficile à satisfaire in concreto, de l'intérieur des pratiques scientifiques, du point de vue d'acteurs ayant incorporé tout un nexus de prescriptions théoriques, axiologiques et pragmatiques. Même si de l'extérieur, pour ceux qui ne sont pas engagés dans les pratiques et en font varier les paramètres in abstracto dans une certaine indifférence, les configurations de départ sont en principe indéfiniment malléables et les solutions aux problèmes en principe infinies.

b) L'argument d'auto-consistance de la physique — qui reste à élaborer - peut être invoqué pour expliquer à la fois le sentiment d'extrême détermination qu'éprouvent les acteurs de la science, et le fait que la possibilité de théories empiriquement équivalentes, irrécusable sur le plan des principes, est de fait si peu fréquemment incarnée dans l'histoire des sciences.

Cela étant dit, est-ce vraiment un argument en faveur de l'inévitabilisme? Une telle explication est-elle en mesure, au terme de chaque phase exploratoire suffisamment poussée d'une recherche, de réduire à l'unité l'ensemble des solutions imaginées tout au long du processus, ou au moins d'imposer une famille restreinte de solutions compatibles entre elles?

La réponse semble être négative, au sens où l'argument d'autoconsistance reste impuissant à éliminer l'éventualité que, dans chaque état de la science, deux physiciens élaborent deux solutions descriptivement incompatibles qui l'une et l'autre satisfassent bien - de l'avis unanime des praticiens - l'ensemble des contraintes partagées qui pèsent sur la recherche, et qui, du coup, apparaissent l'une et l'autre acceptables. 
L'argument reste donc impuissant à bloquer la possibilité d'histoires des sciences incompatibles, parallèles à la nôtre, qui se seraient développées à partir du même état initial mais auraient ensuite manifesté d'autres bifurcations que celles que nous connaissons. Il rend certes la survenue de tels événements très peu probable, du fait de la qualité du ciment descriptif et axiologique commun mis en jeu, mais il ne saurait pour autant les interdire strictement.

En ce point, le réaliste-inévitabiliste s'insurge presque inévitablement contre l'argument d'auto-consistance de la physique. Il proteste contre sa faiblesse, il argue que l'explication de l'inévitabilisme par le réalisme est la seule satisfaisante, car la seule à posséder la force requise.

Et en effet, il semble bien que l'invocation d'une réalité indépendante unique qui est ce qu'elle est, jointe à celle d'une causalité univoque exercée par cette réalité sur l'élaboration scientifique humaine, soit la seule apte à réduire suffisamment les possibles. Seulement, de l'invocation de l'idée à son opérativité dans les pratiques, il y a un grand pas. Répétons le : à examiner ce dont on dispose effectivement dans les pratiques, et même à admettre que des marques non problématiquement données, issues des expériences, représentent la "réalité indépendante ", ces marques ne sont qu'un minuscule point au sein des éléments contraignants qui pèsent sur la recherche, un point dont le réaliste-inévitabiliste est aussi impuissant à démontrer l'action univoquement déterminante que l'argument d'auto-consistance à réduire à l'unité les histoires des sciences possibles.

\section{Conclusion}

Nous espérons avoir convaincu que la charge de la preuve n'est pas plus du côté du contingentisme que de celui de l'inévitabilisme. Nous conclurons sur une remarque importante, qui nous paraît retirer au contingentisme au moins une part du caractère scandaleux que nombre de physiciens sont intuitivement portés à lui associer.

Le contingentisme ne revient nullement à nier que la physique — que notre physique :

- est une pratique effectivement animée d'un souci particulièrement scrupuleux et en acte de vérification de ses propositions;

- est une pratique qui atteint remarquablement cet objectif, au sens où elle établit effectivement des résultats d'une fiabilité remarquable;

- est une pratique qui progresse incontestablement en terme d'adéquation théories-résultats expérimentaux. 
Le contingentisme ne remet pas en cause l'idée que les praticiens sont effectivement soumis à un réseau très dense de contraintes, que ce réseau porte en lui quelque chose qui a droit au titre de Réel, et que tout n'est pas possible ni acceptable. La seule chose que le contingentisme remet en cause, c'est l'idée d'une nécessaire convergence de l'ensemble des histoires des sciences concevables vers un unique ensemble de résultats ${ }^{9}$. Si un tel renoncement apparaît excessif au réaliste correspondantiste, il reste néanmoins parfaitement compatible avec l'affirmation du caractère rationnel de l'activité scientifique et du progrès de la physique.

\section{Bibliographie}

Franklin, Allan. «Experiment in Physics », 1982.

[En ligne] [http:// plato.standford.edu/entries/physics-experiment] (consulté en juillet 2005).

Hacking, Ian. «The Self-Vindication of the Laboratory Sciences ", in A Pickering, Science as Practice and Culture, University of Chicago Press, 29-64, 1992.

- The Social Construction of What?, Harvard University Press, Cambridge, Massachusetts, 1999. (Entre Science et réalité. La construction sociale de quoi?, Paris, La Découverte, 2001).

- - "How Inevitable Are the Results of Successful Science?", Philosophy of Science, 67 (Proceedings), 58-71, 2000.

Hoyningen-Huene, Paul. «Die Wissenschaftsphilosophie Thomas S. Kuhns: Rekonstruktion und Grundlagenprobleme ", Friedr. Vieweg \& Sohn Verlagsgesellschaft mbH, Braunschweig, 1989; traduction anglaise: «Reconstructing scientific revolutions (T. S. Kuhn's philosophy of science)», University of Chicago Press, 1993 ; traduit de l'allemand par A. T. Levine.

Pickering, Andriew. "Constructing Quarks, A Sociological History of Particle Physics ", Chicago, University of Chicago Press, 1984.

—. "The Mangle of Practice. Time, Agency and Science », University of Chicago Press, 1995.

9. La référence à Peirce vient presque inévitablement à l'esprit dans ce genre de discussion, à cause du postulat central fort, chez cet auteur, de l'unicité de la théorie ultime au terme idéal de la recherche : à cause de sa définition de la vérité comme opinion (au singulier) destinée à être acceptée ultimement par tous les chercheurs. Pickering lui-même y fait allusion, dans le contexte de la longue citation invoquée plus haut, à travers une remarque qui identifie le postulat peircien d'unicité de la théorie ultime à une différence essentielle entre le réalisme de Peirce et son propre réalisme pragmatique :

[...] The idea of truth as the future consensus of a community of open-minded inquirers that has dogged pragmatist thought since its inception with Peirce. It seems to me that [...] (our discussion about incommensurability) deprives "future consensus" of the noncontingent associations of uniqueness that such ideas implicitly appeal to. I can imagine future consensuses on all sorts of incommensurable bodies of knowledge, but I cannot imagine how to tell which one of them corresponds to how the world really is (Pickering, 1995, 185, n. 6).

À noter que certains auteurs estiment que la conception de Peirce n'est pas essentiellement incompatible avec l'idée d'une pluralité de théories limites (voir Raïd \& Belabas). 
Raïd Layla et Belabas Karim. "Quine critique de Peirce : vérité et convergence », Philosophi@a Scienti@ae. [En ligne] [http ://philosophiascientiae.free.fr/vol1/raidbel.PDF], 2001.

Sankey, Howard. The Incommensurability Thesis, Aldershot, Avebury, 1994.

Soler, Léna. Introduction à l'épistémologie, Ellipses, 2000a.

—. "Le concept kuhnien d'incommensurabilité, reconsidéré à la lumière d'une théorie structurale de la signification ", Philosophia scientae, 4 (2), 189-217, $2000 \mathrm{~b}$.

—. "The Incommensurability Problem : Evolution, Approaches and Recent Issues ", in L. Soler (dir.), Le problème de l'incommensurabilité, un demi siècle après, Philosophia Scientiae, 8 (1), 1-38, 2004.

- «Une nouvelle forme d'incommensurabilité en philosophie des sciences? ", Revue philosophique de Louvain, août 2006.

- Philosophie de la physique: dialogue à plusieurs voix autour de controverses contemporaines et classiques, entre Michel Bitbol, Bernard d'Espagnat, Pascal Engel, Paul Gochet, Léna Soler et Hervé Zwirn, à paraître chez L'Harmattan, collection «Epistémologie et philosophie des sciences ", 2006a.

- "The Incommensurability of Experimental Practices : the Incommensurability of What? An Incommensurability of the Third-Type ? ", à paraître in L. Soler, H. Sankey and P. Hoyningen, Rethinking Scientific Change and Theory Comparison. Stabilities, Ruptures, Incommensurabilities?, Springer, Boston Studies for Philosophy of Science, 2006b.

Weinberg, Steven. «Reply », New York Review of Books, October, 3, 55-56, 1996. 